\title{
Goldfishing by gauge theory
}

\author{
F. Calogero ${ }^{a, b, 1}$ and E. Langmann ${ }^{c, 2}$ \\ ${ }^{a}$ Dipartimento di Fisica, Università di Roma "La Sapienza", I-00185 Roma, Italy \\ ${ }^{b}$ Istituto Nazionale di Fisica Nucleare, Sezione di Roma \\ ${ }^{c}$ Theoretical Physics, KTH, AlbaNova, SE-106 91 Stockholm, Sweden \\ ${ }^{1}$ francesco.calogero@roma1.infn.it, francesco.calogero@uniroma1.it \\ ${ }^{2}$ langmann@kth.se
}

\begin{abstract}
A new solvable many-body problem of goldfish type is identified and used to revisit the connection among two different approaches to solvable dynamical systems. An isochronous variant of this model is identified and investigated. Alternative versions of these models are presented. The behavior of the alternative isochronous model near its equilibrium configurations is investigated, and a remarkable Diophantine result, as well as related Diophantine conjectures, are thereby obtained.
\end{abstract}




\section{Introduction}

Recently a method has been introduced and exploited to identify new exactly solvable (namely solvable by purely algebraic operations, such as diagonalizing a matrix) many-body problems characterized by equations of motion of Newtonian type ("the acceleration of each particle is determined by the positions and velocities of all particles"), including in particular models of goldfish type (see, for instance, 4, and below). The main idea of this approach - hereafter referred to as the direct method - is to start from an explicitly solvable matrix evolution equation (possibly even quite a trivial one), and to then focus on the time evolution of the eigenvalues of this matrix. For an overview of this method (including an explanation of the terminology used herein), of the main results yielded by it so far, and the quotation of relevant references, we refer to the very recent paper [2].

Another method has been introduced some years ago to treat certain well-know solvable dynamical systems and to illuminate their connection with developments in theoretical particle physics. The main idea of this approach - hereafter referred to as the gauge theory method - is to start from a gauge invariant matrix evolution equation and to exploit the possibility that in one gauge this evolution be trivially simple hence solvable while in another gauge it be related to interesting evolutions, in particular to the equations of motion of Newtonian type of certain many-body problems. For an overview of this approach and the quotation of relevant references, we refer to the relatively recent paper 11. An analysis of the gauge theory approach entailing a clarification of the relation of this method to the direct approach is already provided in the more recent paper published by one of us (EL) [11.

In the present paper, in the context of revisiting this connection, we identify a new solvable many-body problem of goldfish type. This finding hinges on a result obtained many years ago by V. I. Inozemtsev [10]. We also present the isochronous variant of this many-body problem, as well as alternative formulations of these two models, and by investigating the behavior of the alternative isochronous model in the neighborhood of its equilibrium configurations we identify certain remarkable Diophantine relations.

The main new results obtained in this paper are reported in the following Section II. The hasty browser eager to see immediately the equations of motion of the new solvable many-body problems of goldfish type should jump to (11) and for the isochronous variant to (6), and for the alternative versions of these models to (14) and (21); a Diophantine finding and related conjectures are reported at the end of Section II. In Section III the solvable character of the new many-body problems of goldfish type is demonstrated, firstly via the direct method and then via the gauge theory method; the connection among these two approaches is thereby illuminated. In Section IV solvable dynamical systems are derived, which constitute nontrivial alternative reformulations of the many-body problems of goldfish type treated in Section III. In Section V the behavior of the alternative isochronous model in the neighborhood of its equilibrium configurations is investigated and remarkable Diophantine relations are thereby obtained. In Section VI possible future developments are mentioned. The Appendix contains some findings the insertion of which where they are first mentioned (see Remark 2.9 in Section II) would have been too distracting.

\section{Main results}

In this section we report the main new findings obtained in subsequent sections.

The solvable $N$-body problem of goldfish type identified in this paper is characterized by the following equations of motion of Newtonian type:

$$
\ddot{z}_{n}=2 z_{n}\left(z_{n}^{2}-a^{2}\right)+2 \sum_{m=1, m \neq n}^{N} \frac{\left(\dot{z}_{n}+z_{n}^{2}-a^{2}\right)\left(\dot{z}_{m}+z_{m}^{2}-a^{2}\right)}{z_{n}-z_{m}} .
$$

Notation: $z_{n} \equiv z_{n}(t)$ are the dependent variables, $t$ is the independent variable ("time"), superimposed dots denote time-differentiations, $a^{2}$ is an arbitrary constant (we use $a^{2}$ rather than $a$ merely for notational convenience, see below), $N$ is a positive integer (generally we assume $N>1$ ), and indices such as $n, m$ generally take all the values $1,2, \ldots, N$ unless otherwise mentioned.

Remark 2.1. Trivially related models involving additional arbitrary constants could of course be obtained by rescaling the (dependent and independent) variables and by shifting by a constant amount 
the dependent variables; note incidentally that the first factor 2 in the right-hand side of (1) could be changed by rescaling (we put it there for notational convenience, see below), while the second factor 2 (that multiplying the sum) cannot of course be changed. $\square$

Remark 2.2. Although for real $a^{2}$ and for real initial data $z_{n}(0), \dot{z}_{n}(0)$ the time evolution (for real time) of this many-body model entails that the dependent variables $z_{n}(t)$ are as well real, we generally assume the time evolution to take place in the complex $z$-plane (and generally allow the constant $a$ to be as well complex); indeed such an evolution is much more interesting due to the possibility of the "particles" characterized by the complex coordinates $z_{n}(t)$ to go round each other and the related fact that initial data $z_{n}(0), \dot{z}_{n}(0)$ leading to particle collisions are then exceptional (they generally have vanishing dimensionality relative to generic initial data). (If attention is instead restricted to real motions, then the trivial change of dependent variables $z_{n} \rightarrow i y_{n}$ with $y_{n}$ real might be expedient in order to deal with confined motions.) It is possible to reformulate these complex equations of motions as real (and even covariant, even rotation-invariant) equations of motion describing the motion of real point particles in the real (say, horizontal) plane, but we will not take space here to reformulate them in this manner, since the technique to do so is well-known (see for instance Ref. [5]).

The solvable character of these equations of motion is evidenced by the well-known fact [10] [5] that the $N \times N$ matrix evolution equation

$$
\ddot{U}=2 U\left(U^{2}-a^{2}\right)
$$

is itself solvable (in terms of appropriate sigma functions 10]), together with the following

Proposition 2.3. The solution of the initial-value problem for the equations of motion (1) is provided by the following prescription: the coordinates $z_{n}(t)$ are the $N$ eigenvalues of the $N \times N$ matrix $U(t)$ solution of (2) and determined by the following initial data:

$$
\begin{gathered}
U_{n m}(0)=\delta_{n m} z_{n}(0) \\
\dot{U}_{n m}(0)=-\delta_{n m}\left[z_{n}^{2}(0)-a^{2}\right] \\
+\left[\dot{z}_{n}(0)+z_{n}^{2}(0)-a^{2}\right]^{1 / 2}\left[\dot{z}_{m}(0)+z_{m}^{2}(0)-a^{2}\right]^{1 / 2} .
\end{gathered}
$$

Note that the matrix $U(0)$ is diagonal, while the matrix $\dot{U}(0)$ is the sum of a diagonal matrix and a dyadic matrix.

Notation: here and hereafter $\delta_{n m} \equiv \delta_{n, m}$ is the Kronecker delta symbol, $\delta_{n m}=1$ if $n=m, \delta_{n m}=0$ if $n \neq m$.

To obtain the isochronous variant of this many-body problem one starts from the equations of motion

$$
\zeta_{n}^{\prime \prime}=2 \zeta_{n}^{3}+2 \sum_{m=1, m \neq n}^{N} \frac{\left(\zeta_{n}^{\prime}+\zeta_{n}^{2}\right)\left(\zeta_{m}^{\prime}+\zeta_{m}^{2}\right)}{\zeta_{n}-\zeta_{m}}
$$

which correspond to (11) with $a=0$ and with the merely notational replacement of the dependent variables $z_{n}(t)$ with the dependent variables $\zeta_{n}(\tau)$ (and of course now the appended primes denote differentiations with respect to $\tau$ ). One can then apply the procedure usually referred to as "the trick" (see for instance [4] 5]), i. e. (in this case) the following change of dependent and independent variables

$$
\begin{gathered}
\tilde{z}_{n}(t)=\exp (i t) \zeta_{n}(\tau), \\
\tau=i[1-\exp (i t)] .
\end{gathered}
$$

This yields the equations of motion

$$
\ddot{z}_{n}=3 i \dot{z}_{n}+2 \tilde{z}_{n}\left(1+\tilde{z}_{n}^{2}\right)+2 \sum_{m=1, m \neq n}^{N} \frac{\left(\dot{z}_{n}-i \tilde{z}_{n}+\tilde{z}_{n}^{2}\right)\left(\dot{z}_{m}-i \tilde{z}_{m}+\tilde{z}_{m}^{2}\right)}{\tilde{z}_{n}-\tilde{z}_{m}} .
$$

The solution of the initial-value problem is then obviously given by the solution (via Proposition 2.3) of the problem (4) and by the "trick" relations (5), that clearly also imply

$$
\zeta_{n}(0)=\tilde{z}_{n}(0), \quad \zeta^{\prime}(0)=\dot{z}_{n}(0)-i \tilde{z}_{n}(0)
$$


Equivalently, the solution of this model (6) is clearly given by the following

Proposition 2.4. The dependent variables $\tilde{z}_{n}(t)$ that solve the initial-value problem for the Newtonian $N$-body problem (6) are the $N$ eigenvalues of the $N \times N$ matrix $\tilde{U}(t)$ evolving according to the solvable matrix evolution equation

$$
\ddot{\tilde{U}}-3 i \dot{\tilde{U}}-2 \tilde{U}=2 \tilde{U}^{3}
$$

and being moreover characterized by the following initial data:

$$
\begin{gathered}
\tilde{U}_{n m}(0)=\delta_{n m} \tilde{z}_{n}(0) \\
\dot{\tilde{U}}_{n m}(0)=-\delta_{n m}\left[\tilde{z}_{n}^{2}(0)\right] \\
+\left[\dot{\tilde{z}}_{n}(0)-i \tilde{z}_{n}(0)+\tilde{z}_{n}^{2}(0)\right]^{1 / 2}\left[\dot{\tilde{z}}_{m}(0)-i \tilde{z}_{m}(0)+\tilde{z}_{m}^{2}(0)\right]^{1 / 2} .
\end{gathered}
$$

Note that the matrix $\tilde{U}(0)$ is diagonal, while the matrix $\tilde{U}(0)$ is the sum of a diagonal matrix and a dyadic matrix.

The solvable character of the matrix evolution equation (8] is implied by the "trick" formula

$$
\tilde{U}(t)=\exp (i t) U(\tau), \quad \tau=i[1-\exp (i t)]
$$

relating the $N \times N$ matrix $\tilde{U}(t)$ evolving according to (8) to the $N \times N$ matrix $U(t)$ evolving according to (2) with $a=0$.

Remark 2.5. The solvable character [10] of the matrix evolution equation (2) entails that all its solutions $U(t)$ are meromorphic functions of the independent variable $t$. Hence (see (10) all the nonsingular solutions $\tilde{U}(t)$ of the matrix evolution equation (8) are periodic with period $2 \pi$,

$$
\tilde{U}(t+2 \pi)=\tilde{U}(t) .
$$

The singular solutions of (8) are exceptional, corresponding to a set of initial data having vanishing measure with respect to the set of generic initial data.

As an immediate consequence of Proposition 2.4 and of this Remark 2.5 there holds the following

Proposition 2.6. All the solutions of the many-body problem (6) (except those exceptional ones that run into a collision of two or more particles, which correspond to nongeneric initial data) are completely periodic with a period which is a positive integer multiple $p$ of $2 \pi$ :

$$
\tilde{z}_{n}(t+2 p \pi)=\tilde{z}_{n}(t), \quad p=1 \text { or } 2 \text { or...or } N .
$$

The positive integer $p$ accounts for the possibility that the eigenvalues get exchanged among each other through the motion: it depends on the initial data, but it does not change for sufficiently small, if finite, changes of these data and it clearly is not larger than $N$.

This proposition displays the isochronous character of the $N$-body problem ([6), indeed it justifies considering it as one more instance of nonlinear harmonic oscillators [7].

There exists a, by now rather standard, technique to reformulate these type of $N$-body problems, by identifying the $N$ "particle coordinates" $z_{n}(t)$ as the $N$ zeros of a (monic) polynomial in $z$ of degree $N$, and by then focusing on the corresponding time evolution of the $N$ coefficients $c_{m}(t)$ of this polynomial (see for instance [6] [5]):

$$
\psi(z, t)=\prod_{n=1}^{N}\left[z-z_{n}(t)\right]=\sum_{m=0}^{N} c_{m}(t) z^{N-m}, \quad c_{0}=1 .
$$

In Section IV we show how such a procedure is applicable in our case, and we thereby obtain the following alternative formulation of the $N$-body problem (1):

$$
\begin{aligned}
& \ddot{c}_{m}+2(m-1) \dot{c}_{m+1}-2 c_{1} \dot{c}_{m}+2(N+1-m) a^{2} \dot{c}_{m-1} \\
& +(m+2)(m-3) c_{m+2}-2(m-1) c_{1} c_{m+1} \\
& +2\left[m(N+2-m) a^{2}+\dot{c}_{1}-c_{1}^{2}+3 c_{2}\right] c_{m} \\
& -2(N+1-m) a^{2} c_{1} c_{m-1}+(N+2-m)(N+1-m) a^{4} c_{m-2}=0, \\
m= & 1, \ldots, N, \quad c_{0}=1, \quad c_{-1}=c_{N+1}=c_{N+2}=0 .
\end{aligned}
$$


Remark 2.7. The ODE of this system with $m=0$ is identically satisfied; the ODE with $m=N+1$ is also satisfied provided one sets $c_{N+3}=0$, and even the ODE with $m=N+2$ is identically satisfied if one moreover sets $c_{N+4}=0$.

Remark 2.8. A superficial look at this system of ODEs might suggest that it is a linear system of evolution equations for the quantities $c_{m}(t)$; but this is of course not the case, due to the presence of the quantities $c_{1}(t)$ and $c_{2}(t)$. Indeed the highly nonlinear character of this system is already evident by looking at the $N=2$ case, in which case it yields the following (solvable!) fourth order ODE for $f(t) \equiv c_{1}(t)$ :

$$
\begin{array}{r}
f^{\prime \prime \prime \prime} f^{2}-2 f^{\prime \prime \prime} f^{\prime} f^{2}-2 f^{\prime \prime \prime} f^{3}-2\left(f^{\prime \prime}\right)^{2} f+2 f^{\prime \prime}\left(f^{\prime}\right)^{2}+4 f^{\prime \prime} f^{\prime} f^{2}-2 f^{\prime \prime} f^{4} \\
-4\left(f^{\prime}\right)^{2} f^{3}+4 f^{\prime} f^{5}+4 a^{2}\left(f^{\prime \prime} f^{2}-2 f^{\prime} f^{3}\right)=0
\end{array}
$$

(here for typographical convenience differentiations are denoted by appended primes rather than superimposed dots).

Remark 2.9. Two equilibrium (namely, time-independent) solutions of this system (14) are provided by the formula

$$
c_{m}=( \pm a)^{m}\left(\begin{array}{l}
N \\
m
\end{array}\right) .
$$

They are not, however, the only equilibrium configurations of this system. A technique to obtain all these configurations (including this one!) is described in the Appendix.

As indicated above, see (13), the quantities $c_{m}(t)$ that evolve according to the system of ODEs (14) are just the coefficients of the monic polynomial $\psi(z, t)$ of degree $N$ in $z$, the $N$ zeros $z_{n}(t)$ of which evolve according to the equations of motion (11). Hence (see Proposition 2.3) the solution of the system of ODEs (14) is given by the following

Proposition 2.10. The dependent variables $c_{m}(t)$ that solve the initial-value problem for the system of nonlinear ODEs (14) are the $N$ coefficients of the polynomial $\psi(z, t)$, see [13), which is itself given by the formula

$$
\psi(z, t)=\operatorname{det}[z-U(t)],
$$

where the $N \times N$ matrix $U(t)$ evolves according to the solvable matrix evolution equation (2) and is moreover characterized by the initial data (3), with the initial values $z_{n}(0), \dot{z}_{n}(0)$ related to the initial values $c_{m}(0), \dot{c}_{m}(0)$ by the formulas implied by (13),

$$
\begin{gathered}
\prod_{n=1}^{N}\left[z-z_{n}(0)\right]=\sum_{m=0}^{N} c_{m}(0) z^{N-m}, \quad c_{0}=1, \\
-\sum_{n=1}^{N} \dot{z}_{n}(0) \prod_{m=1, m \neq n}^{N}\left[z-z_{n}(0)\right]=\sum_{m=1}^{N} \dot{c}_{m}(0) z^{N-m} .
\end{gathered}
$$

To obtain an alternative version of the isochronous $N$-body problem (6) we use the following version of the "trick":

$$
\begin{gathered}
\tilde{c}_{m}(t)=(-i)^{m} \exp (m i t) \gamma_{m}(\tau), \\
\tau=i[1-\exp (i t)] .
\end{gathered}
$$

Here the quantities $\gamma_{m}(\tau)$ are the dependent variables of the previous model, (14), with $a=0$, up to the (purely notational) change consisting in calling the independent variable $\tau$ (instead of $t$ ) and the dependent variables $\gamma_{m}$ (instead of $c_{m}$ ), so that these variables satisfy the following system of ODEs:

$$
\begin{gathered}
\gamma_{m}^{\prime \prime}+2(m-1) \gamma_{m+1}^{\prime}-2 \gamma_{1} \gamma_{m}^{\prime}+(m+2)(m-3) \gamma_{m+2} \\
-2(m-1) \gamma_{1} \gamma_{m+1}+2\left(\gamma_{1}^{\prime}-\gamma_{1}^{2}+3 \gamma_{2}\right) \gamma_{m}=0 \\
m=1, \ldots, N, \quad \gamma_{0}=1, \quad \gamma_{-1}=\gamma_{N+1}=\gamma_{N+2}=0,
\end{gathered}
$$

where of course appended primes denote differentiations with respect to the independent variable $\tau$ (which we allow to be complex, see (19b). 
Then clearly by applying the "trick" (19) to the system (20) the following new system of nonlinear ODEs is obtained:

$$
\begin{aligned}
& \ddot{\tilde{c}}_{m}+2(m-1) i \dot{\tilde{c}}_{m+1}-\left(2 m+1+2 \tilde{c}_{1}\right) i \dot{\tilde{c}}_{m} \\
& -(m+2)(m-3) \tilde{c}_{m+2}+2(m-1)\left(m+1+\tilde{c}_{1}\right) \tilde{c}_{m+1} \\
& +\left[-m(m+1)+2 i \dot{\tilde{c}}_{1}-2(m-1) \tilde{c}_{1}+2 \tilde{c}_{1}^{2}-6 \tilde{c}_{2}\right] \tilde{c}_{m}=0, \\
& m=1, \ldots, N, \quad \tilde{c}_{0}=1, \quad \tilde{c}_{-1}=\tilde{c}_{N+1}=\tilde{c}_{N+2}=0 .
\end{aligned}
$$

Remark 2.11. The prefactor $(-i)^{m}$ in (19a) is of course unessential, it has been introduced merely to give a marginally nicer look to this system (21) and to some other formulas, see below. With this version, (19), of the "trick" the relation among the particle coordinates satisfying the equations of motion of the isochronous $N$-body problem (6) and the quantities $\tilde{c}_{m}(t)$ satisfying this system of ODEs (21) reads now

$$
\tilde{\psi}(z, t)=\prod\left[z-\tilde{z}_{n}(t)\right]=\sum_{m=0}^{N}(i)^{m} \tilde{c}_{m}(t) z^{N-m}, \quad \tilde{c}_{0}=1,
$$

see (51), (19) and (13). Note that we introduced here the (new) monic polynomial $\tilde{\psi}(z, t)$ having as its $N$ zeros the $N$ dependent variables $\tilde{z}_{n}(t)$ satisfying (6) and as its $N$ coefficients the $N$ dependent variables $\tilde{c}_{m}(t)$ satisfying (21).

This model, (21), is obviously just as solvable as the previous one, (14), indeed the solution of its initial-value problem can be obtained from the solution of the corresponding problem for (14) via the formulas (19) that clearly imply the following relations among the initial data of the two models:

$$
\begin{gathered}
\tilde{c}_{m}(0)=(-i)^{m} \gamma_{m}(0), \\
\dot{\tilde{c}}_{m}(0)-m i \tilde{c}_{m}(0)=(-i)^{m} \gamma_{m}(0) .
\end{gathered}
$$

Equivalently, the solution of this model (21) is clearly given by the following

Proposition 2.12. The dependent variables $\tilde{c}_{m}(t)$ that solve the initial-value problem for the system of nonlinear ODEs [21) are the $N$ coefficients of the polynomial $\tilde{\psi}(z, t)$, see [22), which is itself given by the formula

$$
\tilde{\psi}(z, t)=\operatorname{det}[z-\tilde{U}(t)],
$$

where the $N \times N$ matrix $\tilde{U}(t)$ evolves according to the solvable matrix evolution equation (8) and is moreover characterized by the initial data (9) with the initial values $\tilde{z}_{n}(0), \tilde{z}_{n}(0)$ related to the initial values $\tilde{c}_{m}(0), \dot{\tilde{c}}_{m}(0)$ by the following formulas implied by (22),

$$
\begin{gathered}
\prod_{n=1}^{N}\left[z-\tilde{z}_{n}(0)\right]=\sum_{m=0}^{N}(i)^{m} \tilde{c}_{m}(0) z^{N-m}, \quad \tilde{c}_{0}=1 \\
-\sum_{n=1}^{N}\left[\dot{\tilde{z}}_{n}(0)-i \tilde{z}_{n}(0)\right] \prod_{m=1, m \neq n}^{N}\left[z-\tilde{z}_{n}(0)\right]=\sum_{m=1}^{N}(i)^{m} \dot{\tilde{c}}_{m}(0) z^{N-m} .
\end{gathered}
$$

As an immediate consequence of this Proposition 2.12 and of Remark 2.5 there holds the following

Proposition 2.13. All the nonsingular solutions of the system of ODEs (21) are completely periodic with period $2 \pi$,

$$
\tilde{c}_{m}(t+2 \pi)=\tilde{c}_{m}(t),
$$

while the singular solutions are exceptional, corresponding to a set of initial data having vanishing measure with respect to the set of generic initial data.

This proposition displays the isochronous character of the $N$-body problem (21), indeed it justifies considering it as one more instance of nonlinear harmonic oscillators []. 
Finally, in Section V we obtain all the equilibrium configurations of the isochronous systems (6) and (21) and we study the behavior of the system of nonlinear harmonic oscillators (21) in the neighborhood of its equilibrium configurations. The interested reader will find these results in that section, but we advertise here the Diophantine findings arrived at via this study.

Proposition 2.14. Let the two $N \times N$ matrices $A$ and $B$ be defined componentwise as follows:

$$
\begin{aligned}
A_{n m}= & 2(n-1) \delta_{n+1, m}-\left(2 n+1+2 \bar{c}_{1}\right) \delta_{n, m}+2 \bar{c}_{n} \delta_{1, m}, \\
B_{n m}= & (n+2)(n-3) \delta_{n+2, m}-2(n-1)\left(n+1+\bar{c}_{1}\right) \delta_{n+1, m} \\
& +\left[n(n+1)+2(n-1) \bar{c}_{1}-2 \bar{c}_{1}^{2}+6 \bar{c}_{2}\right] \delta_{n, m} \\
& +2\left[-(n-1) \bar{c}_{n+1}+\left(n-1-2 \bar{c}_{1}\right) \bar{c}_{n}\right] \delta_{1, m}+6 \bar{c}_{n} \delta_{2, m},
\end{aligned}
$$

with the numbers $\bar{c}_{m}$ defined as follows:

$$
\begin{gathered}
\text { for } \nu=0, \quad \bar{c}_{m}=(-)^{m}\left(\begin{array}{l}
\mu \\
m
\end{array}\right), \\
\text { for } \nu=1, \quad \bar{c}_{m}=\delta_{0 m}+\delta_{1 m} \text { if } \mu=1, \\
\bar{c}_{m}=(-)^{m}\left[\left(\begin{array}{c}
\mu-2 \\
m
\end{array}\right)-\left(\begin{array}{c}
\mu-2 \\
m-2
\end{array}\right)\right] \quad \text { if } \mu>1, \\
\text { for } \nu=3, \quad \bar{c}_{m}=(-)^{m}\left[\left(\begin{array}{c}
\mu-3 \\
m
\end{array}\right)+6\left(\begin{array}{c}
\mu-3 \\
m-1
\end{array}\right)+14\left(\begin{array}{c}
\mu-3 \\
m-2
\end{array}\right)+14\left(\begin{array}{c}
\mu-3 \\
m-3
\end{array}\right)\right], \\
\text { for } \nu=4, \quad \bar{c}_{m}=(-)^{m} \sum_{k=0}^{4}\left(\begin{array}{c}
\mu-4 \\
m-k
\end{array}\right)\left(\begin{array}{l}
5 \\
k
\end{array}\right), \\
\text { for } \nu=5, \quad \bar{c}_{m}=(-)^{m}\left[\begin{array}{c}
\mu-5 \\
\left.c\left(\begin{array}{c}
m-5 \\
m-5
\end{array}\right)+\sum_{k=0}^{5}\left(\begin{array}{c}
\mu-5 \\
m-k
\end{array}\right)\left(\begin{array}{l}
5 \\
k
\end{array}\right)\right], \quad c \text { arbitrary . }
\end{array}\right.
\end{gathered}
$$

As indicated above the parameter $\nu$ (the role of which here is mainly to distinguish 5 different cases) can take any one of the 5 values $0,1,3,4,5$, while the parameter $\mu$ can take any positive integer value in the range $\nu \leq \mu \leq N$. Let the $2 N$ numbers $p_{n}^{( \pm)}$be the eigenvalues of the generalized eigenvalue problem

$$
\left(p^{2}+A p+B\right) \underline{r}=0,
$$

(where $\underline{r} \equiv\left(r_{1}, \ldots, r_{N}\right)$ denotes the corresponding eigenvector) implying

$$
\operatorname{det}\left(p^{2}+A p+B\right)=\prod_{n=1}^{N}\left[\left(p-p_{n}^{(+)}\right)\left(p-p_{n}^{(-)}\right)\right] .
$$

Then the $2 N$ numbers $p_{n}^{( \pm)}$are all integers.

Notation: here and throughout the symbol $\left(\begin{array}{l}x \\ y\end{array}\right)$ is the standard binomial coefficient,

$$
\left(\begin{array}{l}
x \\
y
\end{array}\right)=\frac{\Gamma(x+1)}{\Gamma(y+1) \Gamma(x-y+1)} .
$$

We have verified with the help of symbolic programing languages (we used Maple and Mathematica) and for an ample sample of values of $N$ and of the other parameters the validity of this proposition (proven in Section V), and from these computer-aided checks we are led to formulate the following Diophantine conjectures.

Conjecture 2.15. For $\nu=0,1,3,4,5$ and $\mu$ integer in the range $\nu \leq \mu \leq N$ the eigenvalues of the generalized eigenvalue problem (29a) (with 27) and (28)) are given by the following formulas:

$$
\begin{array}{r}
\text { for } \nu=0, \quad \operatorname{det}\left(p^{2}+A p+B\right) \\
=\left\{\prod_{n=1}^{N-\mu}[(p-n)(p-n-1)]\right\}\left\{\prod_{n=1}^{\mu}[(p+n)(p+n-5)]\right\},
\end{array}
$$




$$
\begin{gathered}
\text { for } \nu=1, \quad \operatorname{det}\left(p^{2}+A p+B\right)=(p+1)(p-4) \\
\left\{\prod_{n=1}^{N-\mu}[(p-n)(p-n-5)]\right\}\left\{\prod_{n=1}^{\mu-1}[(p+n)(p+n-7)]\right\}, \\
\cdot\left\{\prod_{n=1}^{N-\mu}[(p-n)(p-n+5)]\right\}\left\{\prod_{n=1}^{\mu-1}[(p+n)(p-n+\mu-7)]\right\} \\
\text { for } \nu=3, \quad \operatorname{det}\left(p^{2}+A p+B\right)=(p+1)(p-4) \\
\cdot\left\{\prod_{n=1}^{N-\mu}[(p-n)(p-n+1)]\right\}\left[\prod_{n=1}^{\mu-4}(p+n)\right]\left[\prod_{n=1}^{\mu}(p+n+1)\right] \\
\cdot\left\{\prod_{n=1}^{N-\mu}[(p-n)(p-n-1)]\right\}\left\{\prod_{n=1}^{\mu}[(p+n)(p-n+\mu-4)]\right\} \\
\left.\qquad \prod_{n=1}^{3}(p-n-1)\right]
\end{gathered}
$$

Here we use the standard convention according to which a product equals unity if the lower limit of the running index exceeds the upper limit.

Remark 2.16. For $\mu=\nu=0$ the validity of this conjecture is certainly true, indeed trivially so (see below the Remark 5.4).

The Conjecture 2.15 only refers to integer values of the parameter $\mu$ in the range $\nu \leq \mu \leq N$. But our computer-aided exploration also indicates the validity, for arbitrary values of the parameter $\mu$, of the following conjecture (which is only formulated below for sufficiently large values of $N$, to avoid less interesting complications).

Conjecture 2.17. The generalized eigenvalue problem (29a) (with (27) and 28a)) features, for arbitrary $\mu$, the $N-1$ eigenvalues

$$
\begin{gathered}
2,3,4,5-\mu, 6-\mu, \ldots, N-\mu, \quad \text { if } \nu=0 \text { or } \nu=5 \text { and } N \geq 5, \\
2,3,4,4-\mu, 5-\mu, \ldots, N-1-\mu, \quad \text { if } \nu=4 \text { and } N \geq 5,
\end{gathered}
$$

and the $N-4$ eigenvalues

$$
\begin{array}{r}
-1,4,6,8-\mu, 9-\mu, \ldots, N-\mu, \quad \text { if } \nu=1 \text { and } N \geq 8, \\
-1,4,6,3-\mu, 4-\mu, \ldots, N-5-\mu, \quad \text { if } \nu=3 \text { and } N \geq 8 .
\end{array}
$$

Remark 2.18. The Conjecture 2.17 - in contrast to the Conjecture 2.15 - does not provide the entire spectrum of the eigenvalue problem (29a), which of course features $2 \mathrm{~N}$ eigenvalues. $\square$

Some aspects of these conjectures are easy to prove. For instance Conjecture $\mathbf{2 . 1 5}$ can be proven by induction for all $N>\mu$ if one assumes its validity for $N=\mu$. But complete proofs of them do not seem quite trivial (see Section VI).

\section{Two proofs of Proposition 2.3}

In this section (part of) the results reported in the preceding Section II are proven, firstly by the direct method, then by the gauge theory method. 


\section{A. Direct method}

The starting point of the direct method is the solvable $N \times N$ matrix evolution equation (2). We then introduce the eigenvalues of the matrix $U(t)$ and the corresponding diagonalizing matrix $R(t)$ via the formulas

$$
\begin{gathered}
U(t)=R(t) Z(t)[R(t)]^{-1}, \\
Z(t)=\operatorname{diag}\left[z_{n}(t)\right],
\end{gathered}
$$

with moreover

$$
R(0)=1
$$

Here and below 1 is the $N \times N$ identity matrix. Note that the first two of these equations, (33a) and (33b), identify (consistently with Proposition 2.3) the $N$ coordinates $z_{n}(t)$ as the $N$ eigenvalues of the $N \times N$ matrix $U(t)$, while the third, (33C), is consistent via the first two with the assignment (3a).

It is then easy to see (for the derivation of these formulas see, if need be, for instance [2]) that, after introducing the $N \times N$ matrix $M(t)$ via the assignment

$$
M(t)=[R(t)]^{-1} \dot{R}(t),
$$

one gets

$$
\dot{U}(t)=R(t)\{\dot{Z}(t)+[M(t), Z(t)]\}[R(t)]^{-1},
$$

entailing (see (330)

$$
\dot{U}(0)=\dot{Z}(0)+[M(0), Z(0)],
$$

as well as the following system of evolution ODEs for the coordinates $z_{n}(t)$ and for the matrix elements $M_{n m}(t)$ of the matrix $M(t)$ :

$$
\begin{gathered}
\ddot{z}_{n}=2 z_{n}\left(z_{n}^{2}-a^{2}\right)+2 \sum_{m=1, m \neq n}^{N}\left(z_{n}-z_{m}\right) M_{n m} M_{m n}, \\
\frac{\dot{M}_{n m}}{M_{n m}}=-2 \frac{\dot{z}_{n}-\dot{z}_{m}}{z_{n}-z_{m}}-M_{n n}+M_{m m} \\
+\sum_{\ell=1 ; \ell \neq n, m}^{N} \frac{z_{n}+z_{m}-2 z_{\ell}}{z_{n}-z_{m}} \frac{M_{n \ell} M_{\ell m}}{M_{n m}}, \quad n \neq m .
\end{gathered}
$$

Note that the time evolutions of the diagonal elements $M_{n n}(t)$ of the matrix $M(t)$ remain unrestricted: it is indeed clear from (33) (implying that $R(t)$ is defined only up to multiplication from the right by an arbitrary diagonal matrix $D(t)$ ) and from (34) that these $N$ functions of time can be chosen arbitrarily without affecting the eigenvalues of $U(t)$, namely the coordinates $z_{n}(t)$. Indeed it is clear that the $N \times N$ matrix evolution equation (2), characterizing the time evolution of the $N^{2}$ matrix elements $M_{n m}(t)$, has now been turned into the system (36), characterizing the time evolution of the $N$ coordinates $z_{n}(t)$ and the $N(N-1)$ off-diagonal elements $M_{n m}(t)($ with $n \neq m)$ of the $N \times N$ matrix $M(t)$.

Clearly this system (36) is no less solvable than the original matrix evolution (2), because its solution can be retrieved from the solution of (2) by purely algebraic operations (essentially, by diagonalizing an $N \times N$ matrix).

But we are interested in obtaining an $N$-body problem involving only the $N$ "particle coordinates" $z_{n}(t)$, hence our next task is to eliminate the $N(N-1)$ "auxiliary quantities" $M_{n m}(t)($ with $n \neq m)$. To do this one must find (assuming it exists) an appropriate ansatz expressing the $N(N-1)$ auxiliary quantities $M_{n m}(t)$ (with $n \neq m$ ) in terms of the $N$ particle coordinates $z_{n}(t)$, taking advantage if need be of the freedom to assign the $N$ quantities $M_{n n}(t)$ at our convenience.

An ansatz that works (in the sense of turning the $N(N-1)$ evolution equations (36b) into identities) is

$$
M_{n n}(t)=-\sum_{\ell=1}^{N} \frac{g}{\left[z_{n}(t)-z_{\ell}(t)\right]^{2}},
$$




$$
M_{n m}(t)=\frac{g}{\left[z_{n}(t)-z_{m}(t)\right]^{2}}, \quad n \neq m,
$$

with $g$ an arbitrary constant. This leads however to an $N$-body model the solvable character of which is already well known [9] [13] [3] [12, hence we do not pursue this development here (we elaborate this point a little further in the following version of the proof).

Remark 3.1. Clearly insertion of this ansatz (37) in the more general matrix evolution equation

$$
\ddot{U}=\Phi(U),
$$

with $\Phi(z)$ an arbitrary scalar function would also work (since this does not depend on the equations of motion satisfied by the coordinates $z_{n}$, see below), and it would lead to the $N$-body problem characterized by the Newtonian equations of motion

$$
\ddot{z}_{n}=\Phi\left(z_{n}\right)-2 \sum_{n=1, m \neq n}^{N} \frac{g^{2}}{\left(z_{n}-z_{m}\right)^{3}} .
$$

This was already noted, many years ago, by Veselov [12. But it appears that, so far, the most general (up to trivial transformations) solvable $N \times N$ matrix evolution of type (38a) is just (2).

Another ansatz that also does (as it were miraculously) work (namely, transform the evolution equations (36b) into identities) reads as follows:

$$
M_{n m}(t)=-\frac{\left\{\left[\dot{z}_{n}+z_{n}^{2}-a^{2}\right]\left[\dot{z}_{m}+z_{m}^{2}-a^{2}\right]\right\}^{1 / 2}}{z_{n}-z_{m}}, \quad n \neq m .
$$

Note that this ansatz, in contrast to the previous one, contains no arbitrary ("coupling") constant $g$. In this case the appropriate assignment for the diagonal elements $M_{n n}(t)$ is quite trivial: $M_{n n}(t)=0$, or equivalently (see (36b) $M_{n n}(t)=\mu(t), \mu(t)$ being an arbitrary function of time (but independent of the index $n$ ). The truth of this assertion can be verified by a trivial if tedious calculation: note that the evolution equations (36a) must also be used in the process.

And it is now clear that the insertion of this ansatz in (36a) yields (11), while its insertion in (35b) yields the assignment (3b). The proof of Proposition 2.3 is thus completed.

\section{B. Gauge theory approach}

Let us now prove again Proposition 2.3, but via the gauge theory method. Although this entails some repetitions we believe it is useful to go through this exercise in some detail, especially because we will now use a somewhat different language - and one purpose of this paper is precisely to clarify the relations among these two different approaches. Moreover this presentation provides some indication of the extent to which this kind of fishing expeditions are likely to yield new goldfishes, namely new interesting solvable models.

Let us start by reviewing (but in a notation more conducive to a direct comparison with the preceding treatment) the gauge theory approach to a more standard model referred to in the literature as $\mathrm{rCM}[1$ [11, characterized by the Newtonian equations of motion

$$
\ddot{z}_{n}=-z_{n}-2 \sum_{m=1, m \neq n}^{N} \frac{g^{2}}{\left(z_{n}-z_{m}\right)^{3}} .
$$

We then present a variant of this approach which leads us to the new solvable $N$-body model of goldfish type presented above.

We start with the following system of dynamical equations for the $N \times N$ matrices $U \equiv U(t), M \equiv$ $M(t)$, and $W \equiv W(t)$ :

$$
\begin{gathered}
\dot{U}+[M, U]=W, \\
\dot{W}+[M, W]=\Phi(U),
\end{gathered}
$$


with the square brackets indicating matrix commutators. As discussed below, to get the rCM model one should assign the function $\Phi(U)$ as follows:

$$
\Phi(U)=-U .
$$

It is however convenient to leave this function $\Phi$ unspecified for the moment; but we require that it contain no other matrix besides $U$, so that $R^{-1} \Phi(U) R=\Phi\left(R^{-1} U R\right)$ for any (invertible) matrix $R$.

The equations (41) have a natural interpretation as gauge theory in $0+1$ dimensions: they are indeed of the form $\left[D_{t}, U\right]=P,\left[D_{t}, P\right]=\Phi(U)$, with $D_{t}=\partial_{t}+M$ being the so-called covariant time derivative with $M$ regarded as gauge field. In particular, they are invariant under the following gauge transformations,

$$
\begin{aligned}
U & \rightarrow \tilde{U}=R^{-1} U R, \quad W \rightarrow \tilde{W}=R^{-1} W R \\
M & \rightarrow \tilde{M}=R^{-1} M R+R^{-1} \dot{R},
\end{aligned}
$$

where the matrix $R \equiv R(t)$ characterizing the gauge transformation is an arbitrarily time-dependent invertible matrix (the transformation rule for $M$ follows from $R^{-1} D_{t} R=\partial_{t}+R^{-1} \dot{R}+R^{-1} M R$ ). One can exploit this invariance to impose additional conditions. In particular for any solution $U(t), M(t)$, and $W(t)$ of (41), one can find a gauge transformation $R(t)$ such that the gauge-transformed matrix $\tilde{M}(t)$, see (43b), vanishes, $\tilde{M}(t)=0$. Indeed, this is implied by the fact that the linear first-order matrix ODE

$$
\dot{R}+M R=0, \quad R(0)=\mathbf{1}
$$

always has a (unique) solution, which can be written as

$$
R(t)=\mathcal{T} \exp \left[-\int_{0}^{t} d s M(s)\right]
$$

where the symbol $\mathcal{T}$ denotes time-ordering. This shows that we can impose the condition

$$
M(t)=0
$$

for all times $t$, without loss of generality. Note that this also implies that, if we impose (45), we will not loose any solution: indeed any solution to our gauge theory equations can be obtained by solving these equations with the condition (45) imposed, and performing a gauge transformation afterwards. In particle physics this latter condition is often called Weyl gauge. We will also use another gauge condition, namely

$$
U_{n m}(t)=\delta_{n m} z_{n}(t)
$$

for all times $t$, which we call diagonal Coulomb gauge. Note that we can impose this latter condition if the matrix $U(t)$ is such that there exists an invertible matrix $R(t)$ such that $R^{-1}(t) U(t) R(t)$ is a diagonal matrix, and this is obviously true in the generic case when the matrix $U(t)$ is non-degenerate. The cases when the matrix $U(t)$ is degenerate correspond to particles in our $N$-body system colliding, and then our solution breaks down (as it should): but this can only happen for exceptional - i. e., nongeneric - initial data, if we allow the particle coordinates $z_{n}(t)$ to move in the complex plane, as we generally do (see the Remark 2.2).

The idea now is that, by imposing the gauge condition (45), we get the matrix equation $\ddot{U}=\Phi(U)$ which might be (chosen to be) exactly solvable, whereas by imposing the condition (46), we get a (hopefully) interesting dynamical system for the variables $z_{n}(t)$. The latter dynamical system can then be solved explicitly as follows: one first determines the solution $U(t)$ of the matrix equation obtained from (41) in the Weyl gauge and with the initial conditions

$$
U_{n m}(0)=\delta_{n m} z_{n}(0), \quad \dot{U}_{n n}(0)=\dot{z}_{n}(0)
$$

Then the eigenvalues of $U(t)$ give the solution of the dynamical system. Note that we can only assign the diagonal elements of $\dot{U}(0)$ since, as we will see, the off-diagonal elements of $\dot{U}(0)$ are determined by another conditions which we have to add. 
Indeed, to get an interesting dynamical system, we need to add one more gauge invariant equation to (411). In particular, to get the rCM model (40), one has to add the equation

$$
[W, U]=J
$$

which is often called Gauss law or momentum map. This latter equation is gauge invariant if the matrix $J=J(t)$ introduced here transforms under gauge transformations as $J \rightarrow \widetilde{J}=R^{-1} J R$. It turns indeed out [1] [11] that if one makes the assignment

$$
J_{n m}(0)=g\left(1-\delta_{n m}\right)
$$

and chooses $\Phi(U)$ as in (42), then the coordinates $z_{n}(t)$ obey the equations of motion of the rCM model (40).

To obtain goldfish type dynamical systems one must instead replace the Gauss' law condition (48) by

$$
B_{n m} B_{n^{\prime} m^{\prime}}=B_{n m^{\prime}} B_{n^{\prime} m}
$$

with

$$
B=W+f(U),
$$

where $f(x)$ is another function to be assigned later. To see that this condition is gauge invariant we note that it can be written as $B \otimes B=\mathcal{P} B \otimes B$ where $\otimes$ is the tensor product (so that $(B \otimes B)(u \otimes$ $v)=(B u) \otimes(B v)$ where $u$ and $v$ are $N$-vectors) and $\mathcal{P}$ is the permutation matrix defined as follows, $\mathcal{P} u \otimes v=v \otimes u$ : the gauge invariance of (50a) follows from the obvious fact that $\mathcal{P}$ commutes with $R \otimes R$.

A new finding (proven below) is then given by the following

Proposition 3.2. The gauge theory equations (41) and (50) are consistent if

$$
\begin{gathered}
f(x)=\alpha+\beta x+\gamma x^{2}, \\
\Phi(x)=\left(\alpha+\beta x+\gamma x^{2}\right)(\beta+2 \gamma x)=f(x) f^{\prime}(x),
\end{gathered}
$$

for arbitrary constants $\alpha, \beta, \gamma$. Imposing the diagonal Coulomb gauge (46]) these equations imply

$$
\ddot{z}_{n}=\Phi\left(z_{n}\right)+\sum_{m=1, m \neq n}^{N} \frac{\left[\dot{z}_{n}+f\left(z_{n}\right)\right]\left[\dot{z}_{m}+f\left(z_{m}\right)\right]}{z_{n}-z_{m}},
$$

and thus the solution of the initial-value problem for this dynamical system can be obtained by solving the corresponding gauge theory equations in the Weyl gauge, see 45 . More specifically: the solution of the initial-value problem for this dynamical system, (52), is given by the eigenvalues of the matrix equation

$$
\ddot{U}=\Phi(U)
$$

with the initial conditions

$$
U_{n m}(0)=\delta_{m n} z_{n}(0)
$$

and

$$
\begin{gathered}
\dot{U}_{n n}(0)=\dot{z}_{n}, \\
\dot{U}_{n m}(0)=\left\{[ \dot { z } _ { n } ( 0 ) + f [ z _ { n } ( 0 ) ] \} ^ { 1 / 2 } \left\{\left[\dot{z}_{m}(0)+f\left[z_{m}(0)\right]\right\}^{1 / 2}, \quad n \neq m .\right.\right.
\end{gathered}
$$

Remark 3.3. In the special case $\gamma=0$ a model is obtained whose solvability was already known 8]. Our new model reported in Section II (and already derived by our other method in the preceding Subsection A) is obtained for $\beta=0, \gamma=1$, and $\alpha=-a^{2}$. But the greater generality of the result as formulated in Proposition 3.2 is only apparent: if $\gamma \neq 0$, one can always reduce this more general case to the special case with $\beta=0$ by the (rather trivial) transformations $U \rightarrow \check{U}=U-\frac{\beta}{2 \gamma} \mathbf{1}, t \rightarrow \check{t}=\gamma t$. And note that, for $f(z)=z^{2}-a^{2}$, the right-hand sides of (55) and (3b) coincide: the apparent differences are merely notational. 
Let us end this section by outlining the proof of this result, whose analogy with that proven in the first part of this section is we trust evident enough not to require further elaboration. We firstly write out (41) and (50) by imposing the condition (45). Then (41a) becomes

$$
\delta_{n m} \dot{z}_{n}+M_{n m}\left(z_{m}-z_{n}\right)=W_{n m}
$$

which for the diagonal elements (i.e. $n=m$ ) implies

$$
W_{n n}=\dot{z}_{n} .
$$

For the off-diagonal elements we obtain the assignment

$$
M_{n m}=-\frac{1}{z_{n}-z_{m}} W_{n m}, \quad n \neq m,
$$

whereas the diagonal elements $M_{n n}$ remain unassigned. Then (41b) reads

$$
\dot{W}_{n m}+\sum_{\ell=1}^{N}\left(M_{n \ell} W_{\ell m}-W_{n \ell} M_{\ell m}\right)=\delta_{n m} \Phi\left(z_{n}\right) .
$$

The diagonal elements of this equation give (via (58) and (57))

$$
\ddot{z}_{n}-2 \sum_{\ell=1, \ell \neq n}^{N} \frac{W_{n \ell} W_{\ell n}}{z_{n}-z_{\ell}}=\Phi\left(z_{n}\right),
$$

while the off-diagonal elements imply the following important consistency conditions,

$$
\begin{aligned}
W_{n m} & +\left(M_{n}-M_{m}\right) \dot{W}_{n m}+\frac{\dot{z}_{n}-\dot{z}_{m}}{z_{n}-z_{m}} W_{n m} \\
& -\sum_{\ell=1, \ell \neq n, m}^{N} W_{n \ell} W_{\ell m}\left(\frac{1}{z_{n}-z_{\ell}}-\frac{1}{z_{\ell}-z_{m}}\right)=0, \quad n \neq m .
\end{aligned}
$$

In particular (50a) implies $B_{n m} B_{m n}=B_{n n} B_{m m}$. Inserting in this equation the assignment $B_{n m}=$ $W_{n m}+\delta_{n m} f\left(z_{n}\right)$ (see (50b) ) and in particular $B_{n n}=\dot{z}_{n}+f\left(z_{n}\right)$ (see (57)), we get

$$
W_{n m} W_{m n}+\delta_{n m}\left[2 \dot{z}_{n}+f\left(z_{n}\right)\right] f\left(z_{n}\right)=\left[\dot{z}_{n}+f\left(z_{n}\right)\right]\left[\dot{z}_{m}+f\left(z_{m}\right)\right] .
$$

Thus the solution of (50) for $n^{\prime}=m$ and $m^{\prime}=n$ is

$$
W_{n m}=\exp \left(\varphi_{n}-\varphi_{m}\right)\left\{-\delta_{n m}\left[2 \dot{z}_{n}+f\left(z_{n}\right)\right] f\left(z_{n}\right)+\left[\dot{z}_{n}+f\left(z_{n}\right)\right]\left[\dot{z}_{m}+f\left(z_{m}\right)\right]\right\}^{1 / 2}
$$

where the functions $\varphi_{n}(t)$ are arbitrary, and it is easy to check that this is a solution of (50) also for all the other values of $n^{\prime}, m^{\prime}$. Inserting this in (60) we obtain

$$
\ddot{z}_{n}=\Phi\left(z_{n}\right)+2 \sum_{\ell=1, \ell \neq n}^{N} \frac{\left[\dot{z}_{n}+f\left(z_{n}\right)\right]\left[\dot{z}_{\ell}+f\left(z_{\ell}\right)\right]}{z_{n}-z_{\ell}} .
$$

It remains to check the consistency relations (61). We note that, for $\ell \neq n, m$ and $n \neq m$, 63) entails $W_{n \ell} W_{\ell m}=W_{n m}\left[\dot{z}_{\ell}+f\left(z_{\ell}\right)\right]$, hence (61) is implied by

$$
\begin{gathered}
\frac{\dot{W}_{n m}}{W_{n m}}=-\left(M_{n}-M_{m}\right)-\frac{\dot{z}_{n}-\dot{z}_{m}}{z_{n}-z_{m}} \\
+\sum_{\ell=1, \ell \neq n, m}^{N}\left[\dot{z}_{\ell}+f\left(z_{\ell}\right)\right]\left(\frac{1}{z_{n}-z_{\ell}}-\frac{1}{z_{\ell}-z_{m}}\right), \quad n \neq m .
\end{gathered}
$$


Inserting the logarithmic derivative of (63) for $n \neq m$ and using (64) we find by straightforward computations that the condition (61) is identically satisfied provided

$$
\dot{\varphi}_{n}=-M_{n}
$$

and the functions $f$ and $\Phi$ satisfy the following functional equations,

$$
f^{\prime}(x)+f^{\prime}(y)=2 \frac{f(x)-f(y)}{x-y}, \quad \Phi(x)=f(x) f^{\prime}(x)
$$

for all $x \neq y$. The general solution of these functional equations is given by (51), and this concludes our proof. Note that at the end we can make the simplifying assignment $\varphi_{n}=M_{n}=0$.

\section{Alternative formulations}

The strategy to obtain alternative formulations of "goldfish-type" $N$-body problems is by now standard (and quite old [6]; for a convenient up-to-date presentation see [2]). One introduces a monic polynomial $\psi(z, t)$ of degree $N$ in $z$, the $N$ zeros $z_{n}(t)$ of which evolve according to the equations of motion of the $N$-body problem under consideration, and then investigates the corresponding evolution of the $N$ coefficients $c_{m}(t)$ of this polynomial. The route we follow to obtain the equations of motions satisfied by the coefficients $c_{m}(t)$ - equations that are of course no less solvable than the equations of motion satisfied by the zeros $z_{n}(t)$, since the relationship among these quantities, the $N$ zeros $z_{n}$ and the $N$ coefficients $c_{m}$ of a polynomial of degree $N$, is purely algebraic - is via the evolution equation satisfied by the polynomial $\psi(z, t)$ : note that this entails that this evolution equation is itself solvable. Since this technique is by now standard, and appropriate formulas to implement it are available (see in particular the Appendix in Ref. [2]), we present without further ado the relevant results.

The evolution equation satisfied by the polynomial $\psi(z, t)$ (see (13)) the zeros of which evolve according to the equations of motion (11) reads

$$
\begin{array}{r}
\psi_{t t}-2\left(z^{2}-a^{2}\right) \psi_{t z}+2\left[(N-2) z-c_{1}\right] \psi_{t} \\
+\left(z^{2}-a^{2}\right)^{2} \psi_{z z}-2\left[(N-3) z-c_{1}\right]\left(z^{2}-a^{2}\right) \psi_{z} \\
+\left\{N(N-5) z^{2}-2(N-2) c_{1} z+2\left[2 N a^{2}+\dot{c}_{1}-c_{1}^{2}+3 c_{2}\right]\right\} \psi=0 .
\end{array}
$$

Notation: here and hereafter subscripted variables denote partial differentiations with respect to them.

Remark 4.1. This evolution equation, [68), contains also certain coefficients $c_{m} \equiv c_{m}(t)$, which are obviously (linearly) related to the function $\psi(z, t)$, indeed clearly (see (13))

$$
c_{m}(t)=\left.[(N-m) !]^{-1} \frac{\partial^{N-m} \psi(z, t)}{\partial z^{N-m}}\right|_{z=0} .
$$

Hence (68) is in fact a nonlinear functional equation satisfied by the polynomial $\psi(z, t)$, and the fact that it is indeed satisfied by a polynomial of degree $N$ in $z$, while not evident, is implied by the way it has been obtained.

From this evolution equation one obtains (using if need be the results in 2]) the corresponding system of ODEs satisfied by the coefficients $c_{m}(t)$, see (14); and this of course justifies the relevant results about the solvability of this system reported in the preceding Section II.

Exactly the same procedure yields (21) from (6), although a more direct route is via the "trick" formula (5), as indicated in the preceding Section II. Anyway we also display here, for completeness, the equation for the polynomial $\tilde{\psi}(z, t)$ (see (22)) that provides the bridge connecting these two systems of ODEs (deriving this equation is particularly easy using the formulas given in the Appendix in Ref. 2]; but beware of the slight notational change in the definition of the coefficients $c_{m}$ due to the $(i)^{m}$ factor in the right-hand side of (22)):

$$
\begin{array}{r}
\tilde{\psi}_{t t}-2 z(z-i) \tilde{\psi}_{t z}+\left[2(N-2) z-(2 N+1) i-2 i \tilde{c}_{1}\right] \tilde{\psi}_{t} \\
+z^{2}(z-i)^{2} \tilde{\psi}_{z z}-2 z(z-i)\left[N(z-i)-3 z-i \tilde{c}_{1}\right] \tilde{\psi}_{z} \\
+\left[N(N-5) z^{2}-2 N^{2} i z-N(N+1)-2(N-2) i \tilde{c}_{1} z-2(N-1) \tilde{c}_{1}\right. \\
\left.+2\left(i \dot{c}_{1}+\tilde{c}_{1}^{2}-3 \tilde{c}_{2}\right)\right] \tilde{\psi}=0 .
\end{array}
$$




\section{Equilibrium configurations, behavior in their vicinity, Dio- phantine relations}

In this section we discuss the equilibrium configurations (namely, the time-independent solutions) of the isochronous models (6) and (21) and the behavior of these models in the vicinity of their equilibria. A motivation for focusing on the isochronous models is that they lead to the remarkable Diophantine relations reported at the end of Section II, as indicated below.

Clearly the equilibrium configuration $\tilde{c}_{m}(t)=\bar{c}_{m}, \dot{c}_{m}(t)=0$ of the system of ODEs (21) is characterized by the following system of $N$ algebraic equations:

$$
\begin{aligned}
& -(m+2)(m-3) \bar{c}_{m+2}+2(m-1)\left(m+1+\bar{c}_{1}\right) \bar{c}_{m+1} \\
& +\left[-m(m+1)-2(m-1) \bar{c}_{1}+2 \bar{c}_{1}^{2}-6 \bar{c}_{2}\right] \bar{c}_{m}=0, \\
& m=1, \ldots, N, \quad \bar{c}_{0}=1, \quad \bar{c}_{-1}=\bar{c}_{N+1}=\bar{c}_{N+2}=0 .
\end{aligned}
$$

Likewise the equilibrium configuration $\tilde{z}_{n}(t)=\bar{z}_{n}, \dot{\tilde{z}}_{n}=0$, of the $N$-body problem (6) is characterized by the following $N$ algebraic equations:

$$
\bar{z}_{n}\left(\bar{z}_{n}-i\right)\left[\bar{z}_{n}+i+\sum_{m=1, m \neq n}^{N} \frac{\bar{z}_{m}\left(\bar{z}_{m}-i\right)}{\bar{z}_{n}-\bar{z}_{m}}\right]=0 .
$$

These two configurations are related to each other by the polynomial formula (see (22))

$$
\bar{\psi}(z)=\prod_{n=1}^{N}\left(z-\bar{z}_{n}\right)=\sum_{n=0}^{N}(i)^{m} \bar{c}_{m} z^{N-m}, \quad \bar{c}_{0}=1
$$

where $\bar{\psi}(z)$ is the "equilibrium" (namely, time-independent) polynomial solution of (70).

The general solution of the algebraic problem (72) can clearly be broken down as follows:

$$
\begin{gathered}
\bar{z}_{n}+i+\sum_{m=1, m \neq n}^{\nu} \frac{\bar{z}_{m}\left(\bar{z}_{m}-i\right)}{\bar{z}_{n}-\bar{z}_{m}}=0 \text { for } n=1, \ldots, \nu, \\
\bar{z}_{n}=i \quad \text { for } n=\nu+1, \ldots \mu, \\
\bar{z}_{n}=0 \quad \text { for } n=\mu+1, \ldots, N,
\end{gathered}
$$

with $\nu$ and $\mu$ nonnegative integers, $0 \leq \nu \leq \mu \leq N$. Of course each of these 3 sectors will be empty if the corresponding range of values of $n$ is empty (recall that $n=1, \ldots, N$ ).

Remark 5.1. In any equilibrium configuration the labeling of the particles can be freely permuted. To write the breakdown (74) we identified, without loss of generality, a (somewhat) definite assignment of particle labels.

Remark 5.2. Genuine equilibrium configurations of the $N$-body problem (6) are characterized by the requirement that $\bar{z}_{n} \neq \bar{z}_{m}$ if $n \neq m$ : indeed, whenever this condition is violated, the equilibrium condition (72) becomes ambiguous due to the vanishing of some denominator in the sum, compensated by a vanishing of the corresponding numerator or by some other cancellation. Hence a necessary condition in order that the configuration associated with the breakdown indicated in (74) correspond to a genuine equilibrium configuration of the $N$-body problem (6) is that $\mu \geq N-1$ and $\nu \geq \mu-1$, so that at most one of the $\bar{z}_{n}$ 's vanishes (in which case we assign to it the highest label, $\bar{z}_{N}=0$ ) and at most one takes the value $i$ (in which case we assign to it the highest or next-to-highest label, $\bar{z}_{N}=i$, or $\bar{z}_{N-1}=i$ if $\bar{z}_{N}=0$ ). But in the following it is convenient to consider all possible equilibrium configurations, including non genuine ones, because, as we will see, such configurations, while problematic to deal with in the context of the $N$-body problem (6), correspond to equilibrium configurations $\bar{c}_{m}$ of the system of ODEs (21) which are instead perfectly legitimate in the context of this nonlinear harmonic oscillators model. Indeed their consideration in such a context yields interesting findings (see below). 
To get more information on the roots $\bar{z}_{n}$ of (74a) we now introduce a monic polynomial $\varphi(z)$ of degree $\nu$ having the $\nu$ numbers $\bar{z}_{n}$ with $n=1, \ldots, \nu$ as its zeros:

$$
\varphi(z)=\prod_{n=1}^{\nu}\left(z-\bar{z}_{n}\right)=\sum_{m=0}^{\nu}(i)^{m} \varphi_{m} z^{\nu-m}, \quad \varphi_{0}=1 .
$$

Note that via this formula we also introduced the $\nu$ coefficients $\varphi_{m}$ of this polynomial.

It is now straightforward (and particularly easy using the formulas given in the Appendix in Ref. 2]; but beware of the slight notational change in the definition of the coefficients $\bar{c}_{m}$ due to the $(i)^{m}$ factor in the right-hand side of (75)) to conclude that this polynomial must then satisfy the following equation, implied by (74a):

$$
\begin{aligned}
& z^{2} \varphi^{\prime \prime}-2(\nu-3) z \varphi^{\prime}+\nu(\nu-5) \varphi \\
= & i\left[z \varphi^{\prime \prime}-2\left(\nu+\varphi_{1}\right) \varphi^{\prime}\right] .
\end{aligned}
$$

It is now easily seen that, via (75), this ODE (176) yields for the coefficients $\varphi_{m}$ the recurrence relation

$$
m(m-5) \varphi_{m}=(m-\nu-1)\left(m+\nu+2 \varphi_{1}\right) \varphi_{m-1},
$$

which must be complemented by the two extremal conditions (see (75))

$$
\varphi_{-1}=\varphi_{\nu+1}=0
$$

and by the normalization condition (see (755)

$$
\varphi_{0}=1 \text {. }
$$

Clearly the two extremal conditions (77b) are identically satisfied (for $m=0$ respectively $m=\nu+1$ ), while the condition (77c) yields (for $m=1$ )

$$
\varphi_{1}=\frac{\nu(\nu+1)}{2(2-\nu)},
$$

entailing the requirement (hereafter assumed to hold)

$$
\nu \neq 2 .
$$

Insertion of (78) in (77a) yields finally the recursion

$$
m(m-5) \varphi_{m}=(m-\nu-1)\left(m+\frac{3 \nu}{2-\nu}\right) \varphi_{m-1},
$$

the solution of which is easily seen to exist only if $\nu \leq 5$. For the remaining cases,

$$
\nu=0 \text { or } 1 \text { or } 3 \text { or } 4 \text { or } 5,
$$

see (79), we get the following solutions (recall (77c)):

$$
\begin{gathered}
\text { for } \nu=0, \quad \varphi(z)=\varphi_{0}=1, \\
\text { for } \nu=1, \quad \varphi_{0}=\varphi_{1}=1, \quad \varphi(z)=z+i, \\
\text { for } \nu=3, \quad \varphi_{0}=1, \quad \varphi_{1}=-6, \quad \varphi_{2}=14, \quad \varphi_{3}=-14, \quad, \\
\text { for } \nu=4, \quad \varphi_{m}=(-)^{m}\left(\begin{array}{c}
5 \\
m
\end{array}\right), \quad m=0,1, \ldots, 4, \\
\text { for } \nu=5, \quad \varphi_{m}=(-)^{m}\left(\begin{array}{c}
5 \\
m
\end{array}\right), \quad m=0,1, \ldots, 4, \quad \varphi_{5} \text { arbitrary } .
\end{gathered}
$$


Via (74) and (75) it is clear that the monic polynomial $\bar{\psi}(z)$ of degree $N$ in $z$, see (73) - which identifies as its $N$ zeros $\bar{z}_{n}$ respectively its $N$ coefficients $\bar{c}_{m}$ the equilibrium configurations of the models (6) respectively (21) - is given by the formula

$$
\bar{\psi}(z)=\varphi(z)(z-i)^{\mu-\nu} z^{N-\mu} .
$$

It is thereby seen, via (82), that the coefficients $\bar{c}_{m}$ are given by the formulas (28) (with the arbitrary constant $c=\varphi_{5}-1$ in (28e), see (82ed).

Remark 5.3. Clearly the coefficients $\bar{c}_{m}$ vanish for $m>\mu$, hence they all vanish (except of course $\bar{c}_{0}=1$ ) if $\mu=0$ (this assignment provides indeed a solution of (71)).

Next, let us discuss the behavior of the system (21) in the neighborhood of its equilibrium configurations. To this end we set

$$
\tilde{c}_{n}(t)=\bar{c}_{n}+\varepsilon \rho_{n}(t)+O\left(\varepsilon^{2}\right)
$$

with $\bar{c}_{m}$ the coefficients $c_{m}$ at equilibrium (as determined above) and $\varepsilon$ a small parameter. We thereby obtain in the standard manner the linearized equations of motion

$$
\begin{array}{r}
\ddot{\rho}_{m}+2(m-1) i \dot{\rho}_{m+1}-\left(2 m+1+2 \bar{c}_{1}\right) i \dot{\rho}_{m} \\
-(m+2)(m-3) \rho_{m+2}+2(m-1)\left(m+1+\bar{c}_{1}\right) \rho_{m+1} \\
+\left[-m(m+1)-2(m-1) \bar{c}_{1}+2 \bar{c}_{1}^{2}-6 \bar{c}_{2}\right] \rho_{m} \\
+2 i \bar{c}_{m} \dot{\rho}_{1}+2\left[(m-1) \bar{c}_{m+1}-\left(m-1-2 \bar{c}_{1}\right) \bar{c}_{m}\right] \rho_{1}-6 \bar{c}_{m} \rho_{2}=0, \\
m=1, \ldots, N, \quad \rho_{0}=0, \quad \rho_{N+1}=\rho_{N+2}=0 .
\end{array}
$$

The general solution of this linear system of ODEs, 855, reads

$$
\rho_{m}(t)=\sum_{n=1}^{N}\left[a_{n}^{(+)} r_{m}^{(+)(n)} \exp \left(i p_{n}^{(+)} t\right)+a_{n}^{(-)} r_{m}^{(-)(n)} \exp \left(i p_{n}^{(-)} t\right)\right],
$$

where the $2 N$ numbers $a_{n}^{( \pm)}$are arbitrary (to be fixed by the initial data) while the $2 N$ numbers $p_{n}^{( \pm)}$, respectively the $2 N$ corresponding ( $t$-independent) $N$-vectors $\underline{r}^{( \pm)(n)} \equiv\left(r_{1}^{( \pm)(n)}, \ldots, r_{N}^{( \pm)(n)}\right)$, are the eigenvalues, respectively the eigenvectors, of the ( $N$-vector) generalized eigenvalue equation (29a). This implies (29b) with the two $N \times N$ matrices $A$ and $B$ defined (componentwise) by the formulas (27). But we know (see Proposition 2.13) that all the nonsingular solutions of the system of nonlinear harmonic oscillators (21) are completely periodic with period $2 \pi$, hence the (certainly nonsingular) solutions describing the behavior of this system around equilibrium must have the same periodicity property, implying that all the eigenvalues $p_{n}^{( \pm)}$yielded by the generalized eigenvalue problem (29a) must be integers. And this entails the validity of Proposition 2.14.

Remark 5.4. In the special case of the equilibrium configuration $\bar{c}_{m}=0$ (for $m=1, \ldots, N$, while of course $\bar{c}_{0}=1$; see the Remark 5.3), the matrices $A$ and $B$ become triangular and the computation of the eigenvalues $p_{n}^{( \pm)}$is then a trivial task, yielding

$$
p_{n}^{(+)}=n+1, \quad p_{n}^{(-)}=n .
$$

\section{Outlook}

It is remarkable that a research project started with the main purpose to clarify a methodological issue - namely, the relationship among two different approaches to the same question: that of identifying solvable many-body problems - resulted in the identification of a novel solvable many-body problem. To the readers who might imagine - in view of the recent discovery of several such new models, as reviewed in Ref. 2] - that this is a relatively trivial task, we suggest to try and find themselves some new model. Our educated guess is that such a task is quite challenging. We are nevertheless ourselves hopeful that new many-body models exist, and that they might be discovered/manufactured by the techniques described in this paper. In any case this possibility remains as a tantalizing prospect, until a way is found to 
ascertain conclusively that these approaches have exhausted their capability to yield many-body models of the kind investigated herein which are both new and interesting (although the second of these two qualities involves of course a value judgment).

Another research direction (perhaps suitable as a $\mathrm{PhD}$ project) is towards proving the Diophantine conjectures proffered in this paper (see the end of Section II) and in previous ones (see [2] and other papers referred to there), as well as obtaining additional findings of this kind (for instance by applying techniques analogous to those of Section $\mathrm{V}$ to the model (14), taking advantage of the results reported in the Appendix).

\section{Acknowledgments}

We gratefully acknowledge financial support by the European Union project RTN "Enigma" and the "Göran Gustafsson Foundation" which made possible a two-week visit to Sweden in February 2006 by one of us (FC) when the results reported in this paper emerged. One of us (FC) would also like to thank the Japan Society for the Promotion of Science (JSPS) and the Yukawa Institute at Kyoto University, in particular Ryu Sasaki, for the pleasant hospitality during a six-week visit to Japan in March-April 2006, when this work was (almost) completed. It is moreover a pleasant duty to acknowledge with thanks the assistance provided to one of us (FC) by Ryu Sasaki by performing some computer-aided checks, by Andrea La Malfa with the handling of Mathematica and by V. I. Inozemtsev with useful suggestions concerning references. One of us (EL) is supported by the Swedish Science Research Council (VR).

\section{Appendix: Equilibrium configurations of the models (14) and (1)}

In Remark 2.9 it was mentioned that there exist additional equilibrium (i. e., time-independent) solutions of the model (14) besides (16). In this appendix we firstly list all these equilibrium configurations (as obtained via Maple) for $N=2,3,4$ and 5 . We then outline a technique allowing to obtain all the equilibrium configurations for arbitrary $N$ (we of course did check that these findings reproduce, for $N=2,3,4,5$, those obtained via Maple).

These equilibrium configurations are clearly solutions of the following set of $N$ algebraic equations (see (14)):

$$
\begin{aligned}
& (m+2)(m-3) c_{m+2}-2(m-1) c_{1} c_{m+1} \\
& +2\left[m(N+2-m) a^{2}-c_{1}^{2}+3 c_{2}\right] c_{m} \\
& -2(N+1-m) a^{2} c_{1} c_{m-1}+(N+2-m)(N+1-m) a^{4} c_{m-2}=0, \\
m= & 1, \ldots, N, \quad c_{0}=1, \quad c_{-1}=c_{N+1}=c_{N+2}=0 .
\end{aligned}
$$

The following solutions of this algebraic system have been obtained via Maple.

For $N=2$

$$
c_{1}=0, \quad c_{2}=-a^{2}
$$

or

$$
c_{2}=\frac{c_{1}^{2}}{3}-\frac{a^{2}}{3}, \quad c_{1} \text { arbitrary }
$$

(the equilibrium configurations (16) correspond to the latter one, (A2b), with $c_{1}= \pm 2 a$ ).

For $N=3$,

$$
c_{2}=\frac{c_{1}^{2}}{3} \pm \frac{a c_{1}}{3}-a^{2}, \quad c_{3}= \pm \frac{a c_{1}^{2}}{3}-\frac{2 a^{2} c_{1}}{3}, \quad c_{1} \text { arbitrary }
$$

(the equilibrium configurations (16) obtain for $c_{1}= \pm 3 a$ ).

For $N=4$,

$$
c_{2}=\frac{c_{1}^{2}}{3}-\frac{4 a^{2}}{3}, \quad c_{3}=-a^{2} c_{1}, \quad c_{4}=-\frac{a^{2} c_{1}^{2}}{3}+\frac{a^{4}}{3}, \quad c_{1} \text { arbitrary }
$$


or

$$
\begin{aligned}
& c_{2}=\frac{c_{1}^{2}}{3} \pm \frac{2 a c_{1}}{3}-2 a^{2}, \quad c_{3}= \pm \frac{2 a c_{1}^{2}}{3}-\frac{5 a^{2} c_{1}}{3}, \\
& c_{4}=\frac{a^{2} c_{1}^{2}}{3} \mp \frac{4 a^{3} c_{1}}{3}+a^{4}, \quad c_{1} \text { arbitrary }
\end{aligned}
$$

(the equilibrium configurations (16) obtain from (A4b) for $c_{1}= \pm 4 a$ ).

For $N=5$,

$$
\begin{aligned}
& c_{2}=\frac{c_{1}^{2}}{3} \pm \frac{a c_{1}}{3}-2 a^{2}, \quad c_{3}= \pm \frac{a c_{1}^{2}}{3}-\frac{5 a^{2} c_{1}}{3}, \quad c_{4}=-\frac{a^{2} c_{1}^{2}}{3} \mp \frac{a^{3} c_{1}}{3}+a^{4}, \\
& c_{5}=\mp \frac{a^{3} c_{1}^{2}}{3}+\frac{2 a^{4} c_{1}}{3}, \quad c_{1} \text { arbitrary }
\end{aligned}
$$

or

$$
\begin{aligned}
& c_{2}=\frac{c_{1}^{2}}{3} \pm a c_{1}-\frac{10 a^{2}}{3}, \quad c_{3}= \pm a c_{1}^{2}-3 a^{2} c_{1}, \quad c_{4}=a^{2} c_{1}^{2} \mp 5 a^{3} c_{1}+5 a^{4} \\
& c_{5}= \pm \frac{a^{3} c_{1}^{2}}{3}-2 a^{4} c_{1} \pm \frac{8 a^{5}}{3}, \quad c_{1} \text { arbitrary }
\end{aligned}
$$

(the equilibrium configurations (16) obtain from (A5b) for $c_{1}= \pm 5 a$ ).

The route we follow to obtain all the solutions of the system (A1) is analogous to that followed in Section V. The starting point is to introduce a monic polynomial $\bar{\psi}(z)$ of degree $N$ that has the $N$ numbers $c_{m}$ solutions of (A1) as its $N$ coefficients:

$$
\bar{\psi}(z)=\prod_{n=1}^{N}\left(z-\bar{z}_{n}\right)=\sum_{m=0}^{N} c_{m} z^{N-m}, \quad c_{0}=1 .
$$

Note the analogy of these formulas with (13), and the fact that we also introduced the $N$ zeros $\bar{z}_{n}$ of this polynomial $\bar{\psi}(z)$, which clearly provide the equilibrium configuration of the $N$-body problem (11) (although not necessarily a genuine equilibrium configuration), hence satisfy the following system of $N$ algebraic ODEs:

$$
\left(\bar{z}_{n}^{2}-a^{2}\right)\left[\bar{z}_{n}+\sum_{m=1, m \neq n}^{N} \frac{\bar{z}_{m}^{2}-a^{2}}{\bar{z}_{n}-\bar{z}_{m}}\right]=0 .
$$

Our strategy to find all the solutions of the system (A1) is to find firstly all the solutions of this system, (A7), and then use (A6).

Clearly the solutions of (A7) can be broken down as follows:

$$
\begin{gathered}
\bar{z}_{n}+\sum_{m=1, m \neq n}^{\nu} \frac{\bar{z}_{m}^{2}-a^{2}}{\bar{z}_{n}-\bar{z}_{m}}=0 \text { for } n=1, \ldots, \nu, \\
\bar{z}_{n}=a \text { for } n=\nu+1, \ldots, \nu+\mu, \\
\bar{z}_{n}=-a \text { for } n=\nu+\mu+1, \ldots, N,
\end{gathered}
$$

with the two nonnegative integers $\nu$ and $\mu$ arbitrary except for the constraint

$$
\nu+\mu \leq N
$$

(implying of course that neither one of these two nonnegative integers can exceed $N$ ).

This assignment, (A8), clearly entails that

$$
\bar{\psi}(z)=(z-a)^{\mu}(z+a)^{N-\mu-\nu} \phi_{\nu}(z),
$$


with $\phi_{\nu}(z)$ the monic polynomial of degree $\nu$,

$$
\phi_{\nu}(z)=\prod_{n=1}^{\nu}\left(z-\bar{z}_{n}\right)=\sum_{m=0}^{\nu} f_{m} z^{\nu-m}, \quad f_{0}=1,
$$

the zeros of which satisfy the algebraic relations A8a . Hence this polynomial $\phi_{\nu}(z)$ satisfies the equation

$$
\left(z^{2}-a^{2}\right) \phi_{\nu}^{\prime \prime}-2\left[(\nu-3) z-f_{1}\right] \phi_{\nu}^{\prime}+\nu(\nu-5) \phi_{\nu}=0,
$$

as implied by the (by now standard) technique to transform algebraic equations such as A8a into differential equations (see for instance the Appendix in Ref. 2]). Here and below primes denote of course differentiations with respect to the argument of the function they are appended to.

Before proceeding to discuss the solution of this equation let us consider the special case with $\nu=0$ entailing $\phi_{0}(z)=1$ (which solves (A10) trivially). In this case (A9a) and (A8d) yield

$$
\bar{\psi}(z)=(z-a)^{\mu}(z+a)^{N-\mu}, \quad \mu=0,1, \ldots, N .
$$

It is then easily seen from (A6) that this entails

$$
c_{m}=a^{m} \sum_{\ell=\max (0, m+\mu-N)}^{\min (\mu, m)}(-)^{\ell}\left(\begin{array}{l}
\mu \\
\ell
\end{array}\right)\left(\begin{array}{l}
N-\mu \\
m-\ell
\end{array}\right) .
$$

This formula provides a set of equilibrium configurations, characterized by the integer $\mu$ in the range $0 \leq \mu \leq N$; in particular the two solutions corresponding to $\mu=0$ and to $\mu=N$ are easily seen to yield the two solutions (16).

Let us now return to (A10), assuming hereafter that the integer $\nu$ is positive, $\nu>0$ (to avoid unnecessary notational complications). To solve this equation, (A10), we set

$$
\phi_{\nu}(z)=a^{\nu} \chi(x), \quad z=a(x-1) .
$$

This formula implies that $\chi(x)$ is again a monic polynomial of degree $\nu$ (although for notational simplicity we do not signal this via a subscript $\nu$ ). We also set (in analogy to (A9b)

$$
\chi(x)=\prod_{n=1}^{\nu}\left(x-x_{n}\right)=\sum_{m=0}^{\nu} \chi_{m} x^{\nu-m}, \quad \chi_{0}=1,
$$

and we then note that this formula, together with

$$
f_{m}=a^{m} \sum_{\ell=0}^{m}\left(\begin{array}{c}
\nu-\ell \\
m-\ell
\end{array}\right) \chi_{\ell},
$$

hence in particular

$$
f_{1}=a\left(\nu+\chi_{1}\right) .
$$

Via this formula and (A12) the differential equation (A10) now reads

$$
x(x-2) \chi^{\prime \prime}-2\left[(\nu-3) x-2 \nu+3-\chi_{1}\right] \chi^{\prime}+\nu(\nu-5) \chi=0,
$$

entailing, via (A13), the two-term recurrence

$$
m(m-5) \chi_{m}=2(\nu+1-m)\left(3-\nu-\chi_{1}-m\right) \chi_{m-1},
$$

implying (for $m=0$ and $m=\nu+1$ ) the extremal conditions $\chi_{-1}=\chi_{\nu+1}=0$ (consistently with (A13)).

For $m=1$ the recurrence formula (A15b) (together with $\chi_{0}=1$, see A13) yields the relation

$$
(\nu-2) \chi_{1}=-\nu(\nu-2),
$$

requiring that the two cases with $\nu=2$ and $\nu \neq 2$ be treated separately. 
For $\nu=2$ one easily obtains the solution

$$
\chi_{0}=1, \quad \chi_{1} \text { arbitrary, } \quad \chi_{2}=\frac{\chi_{1}\left(\chi_{1}+1\right)}{3} .
$$

Remark A.1. Let us note as a curiosity that the recursion (A15b) with $\nu=2$ allows this solution (A17a) to be extended as follows:

$$
\begin{aligned}
\chi_{3} & =\chi_{4}=0, \quad \chi_{5} \text { also arbitrary, } \\
\chi_{m} & =\frac{2^{m-3} 15}{m(m-1)(m-2)}\left(\begin{array}{c}
m-1+\chi_{1} \\
m-5
\end{array}\right) \chi_{5}, \quad m=5,6, \ldots .
\end{aligned}
$$

But we are only interested in the solution A17a with $\chi_{m}=0$ for $m>2$, entailing that $\chi(x)$ is a polynomial of degree $\nu=2$.

So in this $\nu=2$ case we get

$$
\chi(x)=x^{2}+\chi_{1} x+\frac{\chi_{1}\left(\chi_{1}+1\right)}{3}
$$

hence, via A12,

$$
\phi_{2}(z)=z^{2}+f_{1} z+\frac{f_{1}^{2}-a^{2}}{3}, \quad f_{1} \text { arbitrary }
$$

where we set, consistently with A9b $f_{1}=\left(2+\chi_{1}\right) a$.

For $\nu \neq 2$ the recursion (A15b) with A16 yields

$$
\begin{aligned}
\chi_{0} & =1, \quad \chi_{1}=-\nu, \quad \chi_{2}=\frac{\nu(\nu-1)}{3}, \quad \chi_{3}=\chi_{4}=0, \quad \chi_{5} \text { arbitrary, } \\
\chi_{m} & =\frac{(-)^{m-1} 2^{m-3} 15}{m(m-1)(m-2)}\left(\begin{array}{c}
\nu-5 \\
m-5
\end{array}\right) \chi_{5}, \quad m=6, \ldots, \nu .
\end{aligned}
$$

Of course the second line of this equation is only relevant if $\nu>5$, which can only happen if $N>5$.

From these results, via (A9a) with A8d and a12), we arrive finally, after a bit of trivial algebra, at the following two determinations of the polynomial $\bar{\psi}(z)$ (see $\overline{\mathrm{A} 6}$ )):

$$
\begin{gathered}
\bar{\psi}(z)=\left(z^{2}+c z+\frac{c^{2}-a^{2}}{3}\right)(z-a)^{\mu}(z+a)^{N-2-\mu}, \quad \mu=0,1, \ldots, N-2, \\
\bar{\psi}(z)=(z-a)^{\mu}(z+a)^{N-\mu}\left[\begin{array}{c}
\nu a \\
z+a
\end{array}+\frac{\nu(\nu-1) a^{2}}{3(z+a)^{2}}\right. \\
\left.+c \sum_{\ell=0}^{\nu-5} \frac{(-2)^{\ell}}{(\ell+5)(\ell+4)(\ell+3)}\left(\begin{array}{c}
\nu-5 \\
\ell
\end{array}\right)\left(\frac{a}{z+a}\right)^{\ell+5}\right] \\
\nu=5,6, \ldots, N, \quad \mu=0,1, \ldots, N-\nu .
\end{gathered}
$$

The first, A21a of these two formulas is applicable for $N \geq 2$, having been obtained from the previous results corresponding to $\nu=2$, with $c=f_{1}$ an arbitrary number. It includes the results obtainable from the cases with $\nu=1$ and $\nu=3$; likewise, the result corresponding to $\nu=4$ has not be reported, as it is encompassed by the result A11a). The second, A21b), of these two formulas is of course only applicable provided $N \geq 5$, having being obtained from for $\nu \geq 5$, with $c=\chi_{5} / 60$ an arbitrary number. Together with A11a these two formulas determine all the equilibrium configurations $c_{m}$ of the system (14) (and as well all the equilibrium configurations - not necessarily genuine - of the $N$-body problem (11) - up to a final (trivial but tedious) step, to be performed using (A6) (as done above to obtain (A11b) from A11a ), which we leave as a task for the diligent reader. 


\section{References}

[1] Blom J. and Langmann E., "Finding and solving Calogero-Moser type systems using Yang-Mills gauge theories", Nuclear Phys. B 563, 506-532 (1999).

[2] Bruschi M. and Calogero F., "Goldfishing", J. Math. Phys. (submitted to).

[3] Bykovskij B. V. and Inozemtsev V. I., "Elliptic solutions of equations of motion of the interacting particles in an external field", Phys. Lett. A 119, 225-228 (1986).

[4] Calogero F., "The "neatest" many-body problem amenable to exact treatments (a "goldfish"?)", Physica D 152-153, 78-84 (2001).

[5] Calogero F., Classical many-body problems amenable to exact treatments, Lecture Notes in Physics Monograph m66, Springer, Berlin, 2001.

[6] Calogero F., "Motion of poles and zeros of special solutions of nonlinear and linear partial differential equations, and related 'solvable' many-body problems", Nuovo Cimento 43B, 177-241 (1978).

[7] Calogero F. and Inozemtsev V. I., "Nonlinear harmonic oscillators", J. Phys. A: Math. Gen. 35, 10365-10375 (2002).

[8] Calogero F. and Iona S., "Novel solvable extension of the goldfish many-body model", J. Math. Phys. 46, 103515 (2005).

[9] Inozemtsev V. I., "On the motion of classical integrable systems of interacting particles in an external field", Phys. Lett. A 98, 316-318 (1983); "New completely integrable multiparticle dynamical systems", Phys. Scripta 29, 518-520 (1984).

[10] Inozemtsev V. I., "Matrix analogs of elliptic functions", Funct. Anal. Appl. 23, 323-325 (1990) [Russian original: Funct. Anal. Pril. 23, 81-82 (1989)].

[11] Langmann E., "Gauge theory approach towards an explicit solution of the (classical) elliptic Calogero-Moser system", J. Nonlinear Math. Phys. 12 Suppl. 1, 423-439 (2005).

[12] Veselov A. P., "Rational solutions of the Kadomtsev-Petviashvili equation and Hamiltonian systems", Russian Math. Surveys 35, 239-240 (1980) [Russian original: Uspekhi Mat. Nauk 35, 195-196 (1980)].

[13] Wojciechowski S., "On the integrability of the Calogero-Moser system in an external quartic potential and other many-body systems", Phys: Lett. A 102, 85-88 (1984); "On the solvability of the CalogeroMoser system in some external potentials and of other related hamiltonian systems", Phys: Lett. A 104, 189-192 (1984). 